\title{
Soccer shot training system based on CNN
}

\author{
Zheming Yang ${ }^{a}$, Qi Cheng ${ }^{\mathrm{b}}$, Qiuhong Jia ${ }^{\mathrm{c}}$ \\ North China University of Science and Technology, Tangshan 063000, China. \\ a1101121315@qq.com, b937425314@qq.com, c2834281917@qq.com
}

Keywords: Deep learning, CNN, Embedded, Football shot, Train.

\begin{abstract}
The system uses STM32F103 as the main control chip and measures the instantaneous acceleration and angle of the shot by controlling the MPU-6050.Our motion data by hardware to collect a large number of professional players of the instep shot. Based on this data set to train the CNN model we build. We transplanted the trained model to the mobile phone. When the user shoots the door, the device transfers the data to the mobile APP through the HC-05 Bluetooth module. Then the level of shooting is judged by a good model of training so that the pertinent suggestions are made. This system implements the visualization of training data. For a beginner in football, it can help the children to practice effectively. It can also help the coaches to effectively monitor the training of the players and guide the guidance. And it is easy to operate and is suitable for popularization.
\end{abstract}

\section{Introduction}

At present, football is a regular sport in the world, and more and more people begin to pay attention to it. [1] For beginners, only with scientific training can they improve the level of shooting quickly. Often some of the more wealthy students in the family specializes in the intensive training of a football coach. But for a beginner of an ordinary family, the financial burden of private football coaches is relatively large. Even if the beginner is interested in football, the lack of professional training is often not improved.

Deep learning as an important research achievement in the field of brain like computing [2]. At present, convolution neural networks have been widely used in many fields, such as computer vision, Natural Language Processing, information retrieval, speech recognition, semantic understanding and so on. The wave of neural network research has been set off in the industry and academia, which has promoted the development of artificial intelligence [3]. The convolution neural network directly uses raw data as input to automatically learn the representation of features from a large number of training data. Convolution neural network has the characteristics of local connection, weight sharing and pooling operation. It can effectively reduce the complexity of the network, reduce the number of training parameters, and make the model to a certain degree of invariance for translation, distortion and scaling. At present, [4]CNN mainly simulates the complex hierarchical cognition of human brain by increasing the number of layers of the network, using a larger training data set, and improving the existing neural network structure or training learning algorithm. Thus the gap between the human visual system and the human brain is drawn up to enable the machine to obtain the "abstract concept" ability. It has achieved great success in many computer vision tasks, such as [5] image classification, target detection, face recognition, and pedestrians recognition. But in the field of smart wear, it seems that deep learning has not been widely used. Because of the limitation of the data set, our current training effect is not very good. But with our continuous collection of classic shot data, our training will get better and better. At the same time, we also want to start the AI in this field and attract more people to participate in this study. Once it is implemented, it will allow more people to experience the charm of [6]AI. 


\section{System Composition}

\subsection{Hardware Structure.}

The hardware structure is mainly composed of STM32 main control chip, MPU-6050 six axis sensor, and HC-05 Bluetooth module. As shown in Figure 1. The main control chip is STM32F103 produced by ST company. The chip is designed by ST company. It uses a high-performance ARMCortex-M332 bit RISC kernel and its working frequency is $72 \mathrm{MHz}$.It has high speed memory and rich enhanced I/O ports and peripherals connected to two APB buses. ${ }^{[7]}$ It contains 212 bit ADC, 3 common 16 bit timers, and a PWM timer. It also contains standard and advanced communication interfaces, with up to 2 I2C and SPI, 3 USART, one USB, and a CAN. It has a lot better than STC51 in processing speed and function. The full lattice sensing range of the angular velocity of MPU-6050 is $\pm 250, \pm 500, \pm 1000$ and $\pm 2000 \mathrm{dps}$. It can accurately track fast and slow motion and the range of full lattice sensing of accelerator controlled by user program is $\pm 2 \mathrm{~g}, \pm 4 \mathrm{~g}, \pm 8 \mathrm{~g}$ and $\pm 16 \mathrm{~g}$.Product transmission can be transmitted through up to $400 \mathrm{kHz}$ of IIC.MPU-6050 operates under different voltages and its power supply voltage is $2.5 \mathrm{~V} \pm 5 \%, 3.0 \mathrm{~V} \pm 5 \%$ or $3.3 \mathrm{~V} \pm 5 \%$. The size of MPU-6050's packaging is a revolutionary dimension in the industry. So it provides a lot of convenience for our overall design. In addition, we use the HC-05 Bluetooth module to [8] communicate the communication between the device and the mobile phone. It not only provides us with many conveniences, but also greatly reduces the cost.

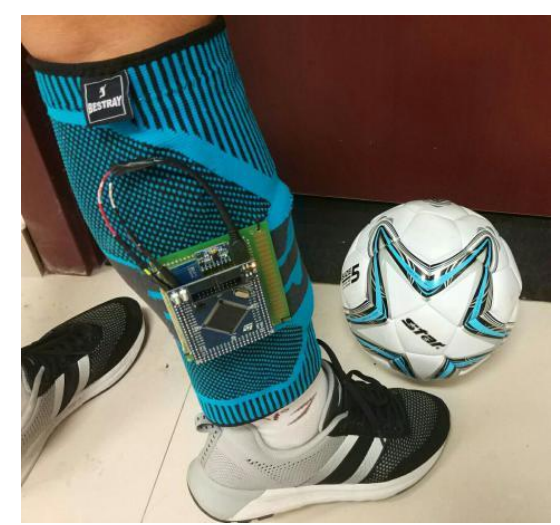

Fig. 1 Hardware design

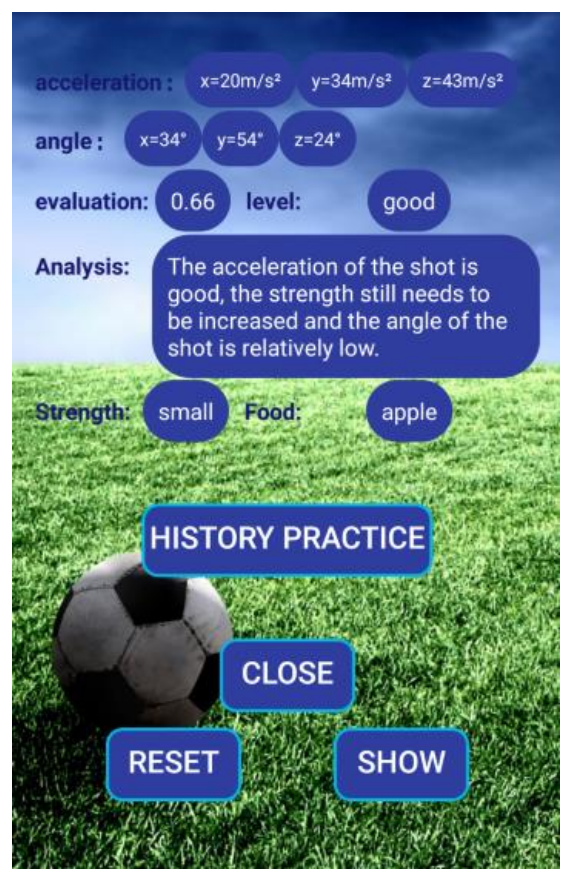

Fig. 2 APP 


\subsection{APP.}

Through APP, we can visualize the data in the shooting process of the sensor to the user. So far, our APP can display the original data, comprehensive evaluation, analysis of advantages and disadvantages, shooting level, exercise, diet and exercise to recommend. As shown in Figure 2.

\section{Algorithm Implementation}

Convolution neural network is a network model for introducing deep learning ideas into neural networks. The process of identifying network training is roughly: First, the convolution is used to perform the operation, and the characteristics of different levels are extracted; Then the network parameters are automatically adjusted by forward propagation, reverse conduction, and gradient descent. Finally, the network model is trained to determine the identification network. In the ordinary multilayer neural network, each layer of nodes is connected to all the nodes of the previous layer. And each connection line has a different weight value and does not share the weight value. Therefore, the values of each layer in each layer are related to all the nodes of the previous layer. Unlike the ordinary multilayer neural networks, the convolution neural network has the characteristics of weight sharing. And in the course of its network training, the nodes of each layer are connected partially. A set of convolution kernel in the convolution neural network generates a feature graph, and the convolution kernel parameters are shared by all nodes on the feature graph. When the network is trained, a set of weights can be obtained. This group of weights can make the network better extract the characteristics of the input and use the classification recognition.

We build a model with two coiling layers, two pool layers, a fully connected layer and one classification layer. We first convert the collected data into a matrix, and then input it into the model. After training we get a criterion for shooting. As shown in Table 1.

Table 1 Training results

\begin{tabular}{cc}
\hline Angle & Effect \\
\hline $0-26.7$ & poor \\
$26.8-48.3$ & good \\
$48.4-57.5$ & excellent \\
$57.6-90$ & poor \\
Acceleration & Effect \\
$0-56.3$ & poor \\
$56.4-113.8$ & good \\
$113.9-162.4$ & excellent \\
$162.5-$ & good \\
\hline
\end{tabular}

\section{Conclusion}

We made a bold attempt. We associate deep learning with shooting training. We have made some improvements in the original model. Although the effect is not very good at present, with the further research, I believe that our accuracy is getting higher and higher. Once it is successful, it will bring convenience to many people.

\section{References}

[1].Hinton G E, Salakhutdinov R R. Reducing the dimensionality of data with neural networks. [J]. Science, 2006, 313 (5786),p.504.

[2].Lecun Y, Boser B, Denker J S, et al. Backpropagation applied to handwritten zip code recognition [J]. Neural Computation, 2014, 1 (4),p.541-551.

[3].Bengio Y, Vincent P, Janvin C. A neural probabilistic language model [J]. Journal of Machine Learning Research, 2003, 3 (6):,p.1137-1155. 
[4].Collobert R, Weston J, Karlen M, et al. Natural Language Processing (Almost) fromScratch [J]. Journal of Machine Learning Research, 2011, 12(1),p.2493-2537.

[5].Le Q V, Mikolov T. Distributed Representations of Sentences and Documents [J]. 2014, 4,p.II1188.

[6].Chen Y. Convolutional Neural Network forSentence Classification [J].2015.

[7].Hinton G E. A Practical Guide to Trainingrestricted Boltzmann machines [J]. Momentum, 2012, 9 (1),p.599-619.

[8].Russakovsky $\mathrm{O}$, Deng $\mathrm{J}$, $\mathrm{Su} \mathrm{H}$, et al. Imagenet large scale visual recognition challenge [J]. International Journal of Computer Vision, 2015, 115 (3),p.211-252.

[9].Lecun Y, Bengio Y, Hinton G. Deep learning. Nature, 2015, 521(7553),p.436-444.

[10].Graves A. Supervised Sequence Labelling with Recurrent Neural Networks [M]. Springer Berlin Heidelberg, 2012.

[11].Kiperwasser E, Goldberg Y. Simple and accurate dependency parsing using bidirectional LSTM feature representations [J]. 2016.

[12].Liu D, Chen T, Liu S, et al. PuDianNao: a polyvalent machine learning accelerator [J]. ACM SIGPLAN Notices, 2015, 43 (1),p.369-381.

[13].Weiss K, Khoshgoftaar T M, Wang D D. A survey of transfer learning [J].Journal of Big Data, 2016, 3 (1),p.9. 